responses is likely to be 'tweakable' through judicious choice of vaccines. In general, extensive molecular studies of SARS-CoV-2 and neutralizing antibody responses will be of value should rational design strategies be needed to generate optimal vaccines ${ }^{12}$.

Overall, we are optimistic, given the number of platforms being investigated and the huge ongoing efforts, that a vaccine (or vaccines) against COVID-19 with immune responses and protection superior to that achieved through natural infection is an achievable goal.

\section{Dennis R. Burton (D) $1,2 \bowtie$ and}

Eric J. Topol(D) 1,3凶

${ }^{1}$ The Scripps Research Institute, La Jolla, CA, USA.

${ }^{2}$ Ragon Institute of MGH, Harvard and MIT,
Cambridge, MA, USA. ${ }^{3}$ Scripps Translational

Research Institute, La Jolla, CA, USA.

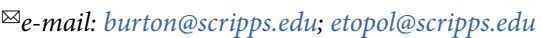

Published online: 30 November 2020

https://doi.org/10.1038/s41591-020-01180-x

References

1. Amanna, I. J. \& Slifka, M. K. Curr. Top. Microbiol. Immunol. 428, $1-30(2020)$.

2. Hammarlund, E. et al. Clin. Infect. Dis. 62, 1111-1118 (2016).

3. Rappuoli, R., De Gregorio, E. \& Costantino, P. Proc. Natl Acad. Sci. USA 116, 14-16 (2019).

$\neg$ 4. Cunningham, A. L. et al. J. Infect. Dis. 217, 1750-1760 (2018).

5. Carter, J. J. et al. J. Infect. Dis. 174, 927-936 (1996).

6. Day, P. M. et al. Cell Host Microbe 8, 260-270 (2010).

7. Cohen, J. Science https://www.sciencemag.org/news/2020/11/

just-beautiful-another-covid-19-vaccine-newcomer-moderna-suc ceeds-large-scale-trial (2020).

8. Klasse, P. J., Nixon, D. F. \& Moore, J. P. Preprints 2020 2020090166 (2020).
9. DeFrancesco, L. Nat. Biotechnol. 38, 1242-1252 (2020).

0. Krammer, F. Nature 586, 516-527 (2020).

11. Lipsitch, M., Grad, Y. H., Sette, A. \& Crotty, S. Nat. Rev. Immunol. 20, 709-713 (2020).

12. Burton, D. R. \& Walker, L. M. Cell Host Microbe 27, 695-698 (2020).

Acknowledgements

D.R.B. receives financial support from The National Institute of Allergy and Infectious Diseases, the Bill \& Melinda Gates Foundation, Ragon Institute, The International AIDS Vaccine Initiative and the Pendleton Trust. Funding for E.J.T. was provided by the Clinical and Translational Science Award (CTSA grant UL1TR002550) from the National Center for Advancing Translational Sciences of the US National Institutes of Health.

D.R.B. and E.J.T. conceived of the idea and wrote the piece together.

\title{
Automated screening of COVID-19 preprints: can we help authors to improve transparency and reproducibility?
}

To the Editor-The COVID-19 pandemic has thrust preprints into the spotlight, attracting attention from the media and the public, as well as from scientists. Preprints are articles not yet published in a peer-reviewed journal, and as such they offer a unique opportunity to improve reporting. The Automated Screening Working Group (https://scicrunch.org/ ASWG/about/COVIDPreprint) aims to provide rapid feedback that may help authors of COVID-19 preprints to improve their transparency and reproducibility.

One quarter of COVID-19 papers published have been preprints. Most of these appear on medRxiv; others appear on bioRxiv or other servers ${ }^{1}$. Although publishing results in preprints allows them to be posted rapidly, the absence of traditional peer review has raised concerns about preprint quality. Unfortunately, it has been impossible for scientists to keep pace with the thousands of COVID-19 preprints published since February. Preprints are vetted before posting to confirm that they describe scientific studies and to prevent posting on topics that could damage public health; however, routine assessment of manuscript quality or flagging of common reporting problems is not feasible at this scale.

Although automated screening is not a replacement for peer review, automated tools can identify common problems. Examples include failure to state whether experiments were blinded or randomized ${ }^{2}$, failure to report the sex of participants ${ }^{2}$ and misuse of bar graphs to display continuous data ${ }^{3}$. We have been using six tools ${ }^{4-8}$ to screen all new medRxiv and bioRxiv COVID19 preprints (Table 1 ). New preprints are screened daily ${ }^{9}$. By this means, reports on more than 8,000 COVID preprints have been shared using the web annotation tool hypothes.is (RRID:SCR_000430) and have been tweeted out via @SciScoreReports (https://hypothes.is/users/sciscore). Readers can access these reports in two ways. The first option is to find the link to the report in the @SciScoreReports tweet in the preprint's Twitter feed, located in the metrics tab. The second option is to download the hypothes. is bookmarklet. In addition, readers and authors can reply to the reports, which also contain information on solutions.

Screening of 6,570 medRxiv and bioRxiv COVID-19 preprints posted before 19 July revealed several interesting results. $13.6 \%$ of preprints shared open data and $14.3 \%$ shared open code, making it easier for others to reuse data or reproduce results. Approximately one third (34.4\%) of COVID-19 preprints acknowledged at least one study limitation. $7.3 \%$ of preprints included bar graphs of continuous data.
This is problematic because many different datasets can lead to the same bar graph, and the actual data may suggest different conclusions from those implied by the summary statistics alone ${ }^{3}$. Therefore, authors should use dot plots, box plots or violin plots instead ${ }^{3}$. Among papers with color maps, $7.6 \%$ used rainbow colormaps, which are not colorblind safe and also create visual artifacts for viewers with normal vision ${ }^{7}$. Rainbow color maps should be replaced with more-informative color maps that are perceptually uniform and colorblind accessible, such as viridis ${ }^{7} .1,775$ preprints (27\%) contained an ethics approval statement for human or animal research. This suggests that nearly three quarters of COVID-19 preprints are secondary or tertiary analyses, modeling studies or cell line studies that do not require approval. Although there are known sex differences in COVID $-19^{10}$, only $20 \%$ of all COVID-19 preprints, and $38 \%$ of preprints with an ethics approval statement, address sex as a biological variable. Statements regarding sample size calculations (1.4\%), blinding $(2.7 \%)$ and randomization (11.4\%) were uncommon, even among studies that contained a human ethics statement (present in $2.4 \%, 5.4 \%$ and $12.6 \%$, respectively). Many COVID-19 preprints are modeling studies, however, and hence these criteria 
Table 1 | Tools used to screen COVID-19 preprints

\begin{tabular}{|c|c|c|}
\hline Tool & Screens for & Link and RRID \\
\hline SciScore $^{4}$ & $\begin{array}{l}\text { Blinding, randomization, sample-size calculations, } \\
\text { sex/gender, ethics and consent statements, } \\
\text { resources, RRIDs }\end{array}$ & http://sciscore.com; RRID:SCR_016251 \\
\hline Limitation- Recognizer ${ }^{6}$ & Author-acknowledged limitations & https://github.com/kilicogluh/limitation-recognizer; RRID:SCR_018748 \\
\hline Barzooka & Bar graphs of continuous data & https://quest-barzooka.bihealth.org; RRID:SCR_018508 \\
\hline
\end{tabular}

asemiautomated tool-requires human confirmation of results. RRID, research resource identifier.

are not always relevant. $6.1 \%$ of preprints used nonhuman organisms, mainly mice. Among the 552 preprints that included cell lines, $7 \%$ described how the cell lines were authenticated (e.g., short tandem repeat profiling) or were kept free of contamination (e.g., mycoplasma detection tests).

Our work shows that it is feasible to conduct large-scale automated screening of preprints and provide rapid feedback to authors and readers. Automated tools are not perfect-they make mistakes, and they cannot always determine whether a problem is relevant to a given paper. Moreover, some problems are too complex for automated tools to detect. Despite these limitations, automated tools can quickly flag potential problems and may complement peer reviews. We hope that these reports will raise awareness about factors that affect transparency and reproducibility, while helping authors to improve their manuscripts. Further research is needed to determine whether automated tools improve reporting.

Tracey Weissgerber (D) 1,2凶, Nico Riedel (D), Halil Kilicoglu ${ }^{3}$, Cyril Labbé4,

Peter Eckmann ${ }^{5,6}$, Gerben ter Riet (D)7,8,

Jennifer Byrne (DD), 90 , Guillaume Cabanac ${ }^{11}$, Amanda Capes-Davis ${ }^{12}$, Bertrand Favier ${ }^{13}$, Shyam Saladi ${ }^{14}$, Peter Grabitz ${ }^{1,2}$,

Alexandra Bannach-Brown?,

\section{Robert Schulz (D) 1,2, Sarah McCann (D) 1,2} Rene Bernard (D) 15 and Anita Bandrowski (iD) 5,6 ${ }^{1}$ Quality $\mid$ Ethics $\mid$ Open Science | Translation (QUEST), Berlin Institute of Health, Berlin, Germany. ${ }^{2}$ Charité-Universitätsmedizin Berlin, Berlin, Germany. ${ }^{3}$ School of Information Sciences, University of Illinois at Urbana-Champaign, Champaign, IL, USA. ${ }^{4}$ University Grenoble Alpes, CNRS, Grenoble INP, LIG, Grenoble, France. ${ }^{5}$ Department of Neuroscience, University of California, San Diego, La Jolla, CA, USA. ${ }^{6}$ SciCrunch Inc., San Diego, CA, USA. ${ }^{7}$ Department of Cardiology, Amsterdam UMC, University of Amsterdam, Amsterdam, the Netherlands. ${ }^{8}$ Urban Vitality Center of Expertise, Amsterdam University of Applied Sciences, Amsterdam, the Netherlands. ${ }^{9} \mathrm{New}$ South Wales Health Statewide Biobank, New South Wales Health Pathology, Sydney, New South Wales, Australia. ${ }^{10}$ Faculty of Medicine and Health, University of Sydney, Sydney, New South Wales, Australia. ${ }^{11}$ UMR 5505 IRIT, Université de Toulouse, UPS, Toulouse, France. ${ }^{12}$ CellBank Australia, Children's Medical Research Institute and The University of Sydney, Westmead, NSW, Australia. ${ }^{13}$ University Grenoble Alpes, TIMC UMR5525, La Tronche, France. ${ }^{14}$ California Institute of Technology, Pasadena, CA, USA. ${ }^{15}$ NeuroCure Cluster of Excellence, Charité-Universitätsmedizin Berlin, corporate member of the Freie Universität Berlin, Humboldt-Universität zu Berlin and Berlin Institute of Health, Berlin, Germany.

$凶_{e-m a i l: \text { tracey.weissgerber@charite.de }}$
Published online: 11 January 2021

https://doi.org/10.1038/s41591-020-01203-7

References

1. Callaway, E. Nature 582, 167-168 (2020).

2. US National Institutes of Health. https://grants.nih.gov/grants/ guide/notice-files/not-od-15-103.html (2015).

3. Weissgerber, T. L. et al. Circulation 140, 1506-1518 (2019).

4. Menke, J., Roelandse, M., Ozyurt, B., Martone, M. \& Bandrowski, A. iScience. 101698 (2020).

5. Riedel, N., Kip, M. \& Bobrov, E. Data Sci. J. 19, 42 (2020).

6. Kilicoglu, H., Rosemblat, G., Malicki, M. \& Ter Riet, G. J. Am. Med. Inform. Assoc. 25, 855-861 (2018).

7. Saladi, S. eLife https://elifesciences.org/labs/c2292989/ jetfighter-towards-figure-accuracy-and-accessibility (2020).

8. Labbe, C., Grima, N., Gautier, T., Favier, B. \& Byrne, J. A. PLoS One 14, e0213266 (2019).

9. Eckmann, P. https://github.com/PeterEckmann1/aswg-pipeline (accessed 15 September 2020).

10. Wenham, C., Smith, J. \& Morgan, R., the Gender and COVID-19 Working Group. Lancet 395, 846-848 (2020).

Acknowledgements

SciScore was funded by the US National Institutes of Health (OD024432, DA039832, DK097771, MH119094, and HHSN276201700124P). The development and application of Seek \& Blastn is supported by grants from the US Office of Research Integrity, grant ID ORIIR180038-01-00 (J.A.B., C.L.), and from the National Health and Medical Research Council of Australia, Ideas grant ID APP1184263 (J.A.B., C.L., A.C.D.). Development of Limitation-Recognizer was partially supported by the intramural research program of the NIH and National Library of Medicine.

Competing interests

A.B. is a cofounder of SciCrunch Inc. 